\title{
Heidegger en torno al error de la Modernidad: La crítica al dualismo cartesiano de cuerpo y alma (Sein und Zeit, parágrafos 19, 20, 21)
}

\author{
Heidegger around Modernity mistake: The critique of the \\ Cartesian dualism of body and soul (Sein und Zeit, \\ paragraphs $19,20,21$ )
}

FERNANDO GILABERT*

\begin{abstract}
Resumen: A partir del pensamiento presentado por Martin Heidegger en Sein und Zeit, podemos elaborar una crítica a la Modernidad filosófica. A nuestro juicio, los presupuestos de esa Modernidad parten del error cartesiano de la dualidad del mundo en dos dominios: la res extensa y la res cogitans, el cuerpo y el alma. A partir de ese error, la filosofía deriva en una ciencia del ente, que no tiene en cuenta al ser, núcleo del pensar filosófico. A partir del concepto heideggeriano de Dasein podemos mostrar como no hay tal dualidad y como es necesario un giro radical en la filosofía.
\end{abstract}

Palabras clave: Heidegger, Modernidad, dualismo, error cartesiano.

\begin{abstract}
As of thought presente by Martin Heidegger in Sein und Zeit, we can develop a critique of philosophical Modernity. In our view, the budgets of the Modernity starts of the Cartesian mistake of the duality of the world in two domains: res extensa and res cogitans, body and soul. From that mistake, philosophy leads to a science of being, which does not considerer the Being, core of philosophical thinking. From Heidegger's concept of Dasein we can show how there is no such duality and as a radical change is necessary in philosophy.
\end{abstract}

Keywords: Heidegger, Modernity, duality, Cartesian mistake.

Fecha de recepción: 02/07/2016. Fecha de aceptación: 20/07/2016.

* Universidad de Sevilla. Doctorando con una tesis acerca de la posibilidad de una teoría política en el pensamiento de Martin Heidegger. Miembro del grupo de investigación de la US Filosofia Aplicada: Sujeto, Sufrimiento, Sociedad (HUM-018). Sus líneas de trabajo son el pensamiento de Martin Heidegger y su relación con la filosofía contemporánea, la filosofía política y el psicoanálisis. Secretario de la revista Differenz. Revista internacional de estudios heideggerianos y sus derivas contemporáneas y del Centro de Estudios Heideggerianos. Entre sus últimas publicaciones encontramos Reacción Opus Clásica, en GADEA, W. F. y ORDÓÑEZGARcíA, J. Democracia y participación política (Hacia una sociedad posible) (2015, Fénix, Sevilla, pp. 57-66) $\mathrm{y}$ The concept of Volk in Heidegger as an exponent of the fundamental ontological structure of Mit-sein, en Diacrítica. Revista do centro do estudos humanisticos (2016, Braga-En imprenta). Contacto: fernando.gilabert. bello@gmail.com 
1. En 1927, al publicarse Sein und Zeit, obra magna de Martin Heidegger, toda la filosofía desde Sócrates a Husserl se tambaleó. No decimos nada nuevo al señalar que, en opinión de Heidegger, los filósofos han olvidado la pregunta esencial de la filosofía, la pregunta por el ser. La tradición filosófica, como señala Heidegger en Sein und Zeit, no se ha preocupado por el ser, y cuando lo ha hecho, ha considerado al ser como un ente, cuando precísamente no puede ser tratado como tal, porque el ser es aquello que determina al ente en cuanto ente (Heidegger, 1977, 6). Considerar al ser como algo obvio implica el riesgo de perder el sentido y, a partir de ello, desarrollar una metafísica que no contemple al ser sino que desarrolle una especulación sobre lo ente conforme a una serie de prejuicios. Esa metafísica especulativa es entonces la contraposición al estudio del ser iniciado en la Antigüedad con el pensamiento presocrático, originando una nueva tendencia en la filosofía basada en lo ente y no en el ser. Este no pensar el ser y centrarse en lo ente es la clave para entender la Modernidad filosófica, contrapuesta al pensamiento antiguo. El origen del abandono del ser y la suplantación del ente como lo relevante en filosofía se encuentra en la propia Antigüedad, en la Grecia clásica que vio nacer el pensamiento presocrático acerca del ser. Tal y como señala Heidegger:

Sobre la base de los comienzos griegos de la interpretación del ser, llegó a constituirse un dogma que no sólo declara superflua la pregunta por el sentido del ser, sino que, además, ratifica y legitima su omisión $(1977,2)$.

La característica fundamental de la Modernidad filosófica es entonces el estudio del ente y el olvido del ser. Desde entonces, toda la tradición contribuye a ese olvido. Y aunque la filosofía escolástica medieval estudió el ser, lo expuso también como si fuera un ente. Pero en el siglo XVII, en plena Edad Moderna, la pregunta cambia en su totalidad, y con ello el estudio más representativo de la filosofía, que ahora será el estudio del conocimiento, la epistemología.

Del XVII se ha dicho que es el siglo de la física por la importancia de los descubrimientos que para esta disciplina en él tuvieron lugar. Este hecho no es banal para nuestra investigación: la física es la ciencia que estudia al ente en su particularidad espacial y material, obviando al resto de entes. El predominio de la física desde esta centuria implica la confusión del ente con el ente material, aislando, por no poder probarse físicamente, al ente no material. Esos entes que escapan del dominio de la física no son el objeto de la filosofía moderna, sino que ésta tiene, como señalamos, como objeto de su estudio la epistemología, la teoría del conocimiento, el modo que tiene el hombre de aprehender los entes materiales, los cuales se encorsetan en unos parámetros según los que, o bien se consideran como provistos de materialidad, esto es, buscando los nexos de la imaginación con la física mediante procesos neuronales y estimulos fisioquímicos, o bien, como aquello que aporta el hombre para el proceso del conocimiento. Pero a pesar de su modernidad, la

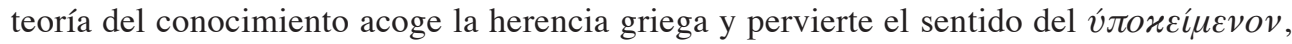
"lo que subyace", y que en la Metafísica aristotélica era algo que se predicaba de otras cosas pero no de otras personas (Aristóteles, 1996, II, 1), transformándose en la Edad de la Razón en sujeto, del latín subjectum. Este sujeto se convierte en el centro de la reflexión filosófica moderna, y, por ende en el centro del Universo. De ahí que la Modernidad filo- 
sófica concentre sus esfuerzos en vincular la relación del hombre-sujeto con la realidad que le rodea. El mundo físico-material es la realidad que rodea al hombre, núcleo cohesionador de esa realidad, la cual se convierte en un binomio donde el hombre particular e individual, identificado con el sujeto, trata de aprehender esa realidad, aquellos entes materiales que conforman lo que se llama mundo. Este mundo ajeno al sujeto se compone de cosas, los objetos, que rodean al sujeto, siendo el mismo mundo una cosa más. Así, sujeto y objeto son los puntos de referencia de la teoría del conocimiento de la filosofía moderna, girando siempre la pregunta filosófica en torno a ese binomio, inclusive en el tema que pretextamos para nuestro trabajo, el dualismo cuerpo y alma. El leitmotiv de la cuestión filosófica moderna es la aprehensión del objeto por parte del sujeto, la cual siempre será analizada siguiendo las pautas de la ciencia moderna, cuya disciplina más representativa es la física, no contemplándose en ningún momento a la filosofía como base de la ciencia, sino que más bien se trata de encorsetar a la filosofía como si de una ciencia más se tratara, siguiendo modelos físicos o matemáticos.

Podría parecer que Heidegger como fenomenólogo estaría en esa línea. Husserl, el padre de la fenomenología y maestro de Heidegger, es continuador de la Modernidad en el doble sentido de que quiere considerar a la filosofía como ciencia estricta y de que su interés radica en ir a las cosas mismas, al objeto puro (Moreno Márquez, 2000). Pero Heidegger se aparta del camino trazado por la fenomenología husserliana, tomando de ésta el método pero apartándose radicalmente del binomio sujeto-objeto, roto al emplear el término Dasein, donde sujeto y objeto no son una dualidad porque la conciencia de sí mismo (identificable con el sujeto) y el mundo (identificable con el objeto) se dan indisolublemente al constituirse en estructuras fundamentales de dicho Dasein.

2. El giro hacia el sujeto y el olvido del ser en el XVII tiene como uno de sus principales puntas de lanza la filosofía de Descartes, quien es además uno de las principales figuras de la revolución científica. Para Heidegger todo el entramado de la filosofía cartesiana es un error que ha perjudicado seriamente a todo el pensamiento posterior al racionalismo debido a la herencia centrada en el planteamiento con el que Descartes busca establecer el edificio del saber. En el apartado B del capítulo tercero de la primera sección de la primera parte de Sein und Zeit, en los parágrafos 19, 20 y 21, Heidegger confronta su teoría con la de Descartes (Heidegger, 1977, 89 y ss.). No será la única vez: esta confrontación está presente en todo el camino del pensar heideggeriano, como en los estudios sobre Nietzsche (Heidegger, 1997) o en los seminarios de Zollikon (Heidegger, 2006), donde ataca una y otra vez al racionalismo cartesiano. Nos centraremos en Sein und Zeit, porque creemos que ahí se hallan las bases de la oposición de Heidegger a la filosofía cartesiana, sobre todo en relación a la cuestión del dualismo. Esta batalla librada contra Descartes en Sein und Zeit, clarifica que la interpretación heideggeriana de la mundaneidad es más acertada que la concepción cartesiana del mundo. A ese respecto, Heidegger señala que

con el cogito sum, Descartes pretende proporcionar a la filosofía un fundamento nuevo y seguro. Pero lo que en este comienzo radical Descartes deja indeterminado es el modo de ser de la res cogitans, más precísamente el sentido de ser del sum $(1977,24)$. 
En Descartes, el mundo no es un mundo donde el binomio sujeto-objeto no tiene sentido al fundirse en el horizonte de la estructura fundamental del Estar-en-el-mundo (In-der-Weltsein) como sucede en el planteamiento heideggeriano, sino que el punto de partida es que los objetos que hay en este mundo dan una imagen que, a juicio de Heidegger, no tiene que ver con el mundo mismo como fenómeno. Para Heidegger, "la interpretación (usual) del mundo toma su punto de partida primeramente en los entes intramundanos, y termina por perder de vista el fenómeno del mundo" (Ib, p. 89). Cuando Heidegger habla aquí de interpretación usual del mundo no se refiere sólo al cartesianismo, también hace referencia al pensamiento post-socrático y medieval que había dado preeminencia a lo óntico, los entes, sobre lo ontológico, el ser (Ib., 8-15).

Aunque Heidegger critica la visión que sobre el ser se tiene tras la irrupción del platonismo en la historia de la filosofía, piensa que es aún peor la visión surgida con Decartes, entre otras cosas, por corromperse la visión de los postaristotélicos y originando una ontología fallida que parte de una ontología anterior también fallida donde se secularizan términos teológicos en términos antropológicos, sin sentido en dicha disciplina, entre otras cosas, porque sitúan al hombre en la categoría de lo divino, una categoría ajena a lo humano. Al respecto, en una conferencia de 1924 que allana el camino para lo que en 1927 será Sein und Zeit, Heidegger señaló que la terminología teológica no puede derivar en una terminología filosófica porque el cometido de teología y filosofía es diferente $(2004,107)$.

$\mathrm{Si}$, según Heidegger, la filosofía griega y medieval yerran en sus pretensiones al cometer un error de fundamentos, la filosofía post-cartesiana, alzada sobre estos fundamentos equivocados, construye un error sobre el error, un equívoco cuya base está en Descartes y que Heidegger denuncia minuciósamente con ocasión de su exposición de la mundaneidad en Sein und Zeit.

3. La tradición filosófica nos ha dejado un buen puñado de críticas a la teoría de Descartes, si bien el cartesianismo ha tenido cierta entidad como escuela racionalista, en muchos de sus casos el trabajo de sus continuadores ha sido enmendar los errores habidos en la teoría del "padre de la Modernidad", sobre todo los relativos a su concepción dualista (Ryle, 2000). Esa misma dualidad es la base de la crítica de Heidegger, pero entre las críticas de la tradición y la de Heidegger hay diferencias notables en función de los intereses filosóficos de cada una.

La concepción dual que Descartes tiene del mundo y del hombre se despliega en las Meditationes de prima philosophia y en los Principia Philosophiae sobre todo. Allí formula que el hombre es un ser dotado de cuerpo y alma y que esta dualidad tiene su correlato en el mundo, siendo el alma o res cogitans lo pensante y el cuerpo o res extensa lo material. Estas obras serán el blanco de la crítica que Heidegger hace en Sein und Zeit. Según nos indica Heidegger

Descartes ve la determinación fundamental del mundo en la extensio. Y dado que la extensión es un momento constitutivo de la espacialidad y que, según Descartes, es incluso idéntica con ella, y que, por otra parte, la espacialidad es en cierto modo constitutiva del mundo, la discusión de la ontología cartesiana del mundo nos depara, al mismo tiempo, un punto de apoyo negativo para la explicación positiva de la espacialidad circundante y del Dasein mismo $(1977,89)$. 
Con esto, Heidegger nos muestra que su crítica a Descartes viene por el materialismo con el que el francés toma al mundo, tratándolo como algo ajeno sin entidad propia salvo un compendio de entes. Descartes no se preocupa por el mundo en su conjunto, dándolo por sentado como algo obvio, como un conjunto de objetos, mera extensión constituída por cuerpos (objetos, entes) que están en un espacio. La res cogitans, el alma, está "por otro lado". Entonces, según el planteamiento cartesiano, el sujeto no habita este mundo de objetos, sino que está más allá de lo corpóreo, estando, como sucedía en el platonismo, encerrado en un cuerpo material (Platón, 1989). De igual modo sucede con el mundo: el mundo material es extensión, existiendo un ser pensante ajeno a dicho mundo que podría identificarse con la divinidad. Pero para Heidegger no existe la posibilidad de contemplar tal dualidad porque la conciencia propia de sí y el mundo están ligados indisolublemente, puesto que el Dasein tiene como una de sus estructuras fundamentales el Estar-en-el-mundo (In-der-Welt-sein).

Así, para Heidegger, distinguir entre cosa pensante y cosa material, como hace Descartes, es disolver lo indisoluble. Es más, la distinción cartesiana de alma y cuerpo implica diferenciar entre espíritu y naturaleza, hacerlos opuestos, enfrentarlos. Pero esta oposición está falta de fundamentos a pesar de todas las variaciones que ha tenido esta idea a partir del planteamiento cartesiano y aunque desde entonces prevalezca la oposición entre sujeto y objeto como dos cosas desvinculadas entre sí, como dos mundos ajenos, a saber, un mundo espiritual, vinculado al alma, y un mundo material, cuyo ser propio es la naturaleza. Y así, de Descartes a Hegel, la Modernidad se ha basado en el error de la dualidad al considerarla obvia. Para Heidegger

por múltiples y variadas que sean las modulaciones ónticas que fijan el contenido de esta pareja de conceptos opuestos, la falta de claridad de sus fundamentos ontológicos y la de las partes antagónicas mismas tienen su raíz inmediata en la distinción hecha por Descartes $(1977,89)$.

Por ello, Heidegger se plantea en qué comprensión del ser se basa Descartes para constituir esta idea de dualidad, hallando la respuesta en la confusión cartesiana del término sustancia al aplicarlo a lo ente, por lo que ser y ente se dan en conjunto como una y la misma cosa:

El término para el ser de un ente que es en sí mismo es el de substantia. Esta expresión mienta tanto al ser del ente que es sustancia, vale decir, la sustancialidad, como el ente mismo: una sustancia (Ib., 89-90).

Es curioso como Descartes, aquél del que criticamos la dualidad, precísamente su mayor error haya sido unificar bajo el mismo concepto de substancia la diferencia que hay entre ser y ente. Con eso, configuramos que el error cartesiano es la dualidad entre lo pensante y lo corpóreo y, por otro lado, la sustancialidad de lo corpóreo.

4. Una explicación se presenta como necesaria sobre la consideración que Descartes hace de la extensión en base a "la sustancialidad de esta res corpórea entendida como sustancia" (Ib., 90). En la tradición, la noción de sustancia viene dada a partir de Aris-

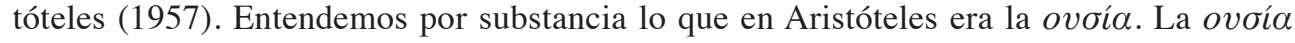


puede definirse como "lo que es" pero se traduce como "lo que subyace", como se

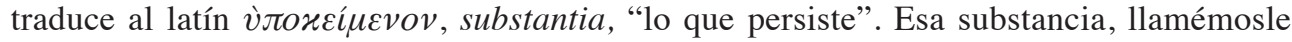
así de manera canónica, tenía en Aristóteles diversas interpretaciones (1957, L, VII, I), destacando la de substancia material y la de substancia primera, que se vinculan en la tradición filosófica al estudio del ser. La substancia material aristotélica es el equivalente cartesiano de lo corpóreo y extenso, ya que la extensión se asocia a la materia, mientras que la res cogitans se relaciona con lo espiritual, no material. Ahora bien, en Aristóteles la substancia primera era el ser, aquello que existe en el mundo y de lo que se podría predicar cualquier cosa, los atributos (1957, L, VII, I). Heidegger y Aristóteles presentan al ser como la substancia primera, dejando aparte a unas "substancias segundas" que, siguiendo las pautas del estagirita, serían los accidentes, identificados con los atributos, lo que atribuímos a los existentes, a la substancia. Pero en Descartes, al dar por hecho que ser, la substancia, y ente, su substancialidad, son lo mismo, se yerra el tiro que desorienta a todo el pensamiento moderno.

Heidegger señala que "las substancias son accesibles en sus atributos, y cada substancia tiene una propiedad característica en la que se puede encontrar la esencia de la substancialidad de esa determinada substancia" (1977, 90), esto es, que el ser de las cosas se muestra oculto, siendo la manera de desvelarlo aquello que le es propio, su fenómeno más característico. Este fenómeno propio de cada cosa es lo que permite al ser desvelarse, lo que permite a la substancia ser tal substancia. Pero en Descartes, la res corpórea posee propiedades que implican una especie de ser antes que el ser: Afirma que lo que le es propio a la res corpórea es "la extensión a lo largo, ancho y profundo" (1974). Esto es, se dota a la substancia con atributos pero queda indefinido lo propio de ella, no se define aquello que lo hace substancia, sino que se le otorgan una serie de cualidades, como si de alguna manera ya tuviera ser antes de dotarlo de ser, como si ya estuviese antes de ser. Según Heidegger, para Descartes "la extensión es aquella estructura del ser del ente en cuestión, que ya tiene que ser antes de todas las demás determinaciones de ser para que éstas puedan ser lo que son" (1977, 90). Ello implica que el espacio que ocupan las cosas ya se da previamente a las cosas mismas, no pudiendo entonces demostrarse que aquello que es el mundo tenga como atributo principal el espacio que ocupe, su extensión misma, porque implicaría que hay mundo antes del mundo, algo anterior a cualquier aspecto de éste, dejando a esa extensión al margen de toda comprensión al darse por hecho. Para Heidegger

la demostración de la extensión y de la substancialidad del mundo caracterizada por aquella se realiza mostrando como todas las demás determinaciones de esta substancia, y en particular las de divisio, figura y motus, sólo pueden concebirse como modi de la extensio y que, a la inversa, la extensio resulta comprensible sine figura vel motu $(I b ., 90)$.

Con ello, la cosa en tanto que corpórea puede variar de muchos modos siempre que mantenga su extensión, esto es, que mediante el movimiento puede ser de muchas maneras, quedando siempre algo que permanece, el ser, el cual, como vemos, es relacionado por Descartes con la extensión. 
5. Pero no sólo de extensión vive el error cartesiano. Relacionar ser y extensión no es el único desacierto de la teoría de Descartes, también el otro aspecto de ese dualismo del que está impregnada toda su filosofía presenta el problema de la unicidad entre substancia y substancialidad al dar por hecho que ambas son lo mismo en relación a la res extensa. Desde el planteamiento cartesiano "por substancia no podemos entender sino un ente que es de tal manera que para ser no tiene necesidad de otro ente" (Ib., 92). Esto implica que la substancia es independiente y no creada, no dependiendo de nada más allá de ella misma, lo cual estaría dentro de la definición de mundo como extensión al vincularse a la substancia. Pero "el ser de una substancia se caracteriza por una no-necesidad" (Ib., 92), matizando así la idea de independencia que tiene consigo el ser con respecto del ente en la teoría cartesiana. Así, tenemos entonces que, para Descartes, el ser de las cosas materiales, de lo extenso, es independiente y no creado. A este respecto nos sale al paso la pregunta de si Descartes viene a decirnos con esto que este mundo material existe desde siempre, siendo eterno y por tanto reuniendo las características propias del ens perfectissimus, de lo divino, de Dios. La respuesta es un no rotundo. El propósito cartesiano, según lo ve Heidegger y de ahí parte su crítica, es totalmente al contrario: Dios es el creador y el mundo material es lo creado. El mundo material depende entoces de Dios.

Con esto topamos con la diferencia entre lo eterno y lo perecedero (lo temporal), entre lo infinito y lo finito. Esta incursión en la teología posee relevancia ontológica al haber entre lo infinito y lo finito una diferencia notable en el grado de ser de uno y otro. Se da por hecho que Dios, el ens perfectissimus, y el mundo, el ens creatum, son entes y que como tales albergan en su interior una noción de ser. Pero al tener uno como propiedad la infinitud y otro la finitud, al ser uno infinito y el otro finito, damos al término "ser" un sentido tan amplio que llega al infinito. Por ello, podemos señalar que según Descartes, lo creado, el mundo finito, alberga algo independiente pero, en cierto modo, "creado" al ser finito: los dos términos de la dualidad cartesiana, la res cogitans y la res extensa, pensamiento y extensión, alma y cuerpo, que son finitas pero participan de lo infinito al ser productos de lo divino.

Entonces, para aclarar qué es la substancia se necesitará saber qué es lo que comparten la divinidad, que es infinita, con la res cogitans y la res extensa, finitas como decimos. Para Heidegger

el ser de la substancia cuya proprietas distintiva es la extensio será ontológicamente determinable, en principio, cuando se haya aclarado el sentido de ser que es común a las tres substancias, a la infinita y a las dos finitas (Ib., 92-93).

Pero Descartes omite este problema, no sabemos si intencionalmente para cuadrar su sistema o porque no reparó en ello. Heidegger advierte que este problema fue ampliamente debatido por la escolástica cuando trató de relacionar la finitud con la infinitud al mostrarse las diferencias entre el ser infinito divino y el ser finito de lo mundano. Pero "al eludir este problema, Descartes deja sin examinar el sentido del ser involucrado en la idea de substancialidad y el carácter de universalidad de esta significación" (Ib., 93).

Descartes no sólo elude completamente la pregunta ontológica por la substancialidad, sino que también afirma explícitamente que la substancia como tal, es decir, la substancialidad es inaccesible en sí misma $(I b ., 94)$. 
Por ello, todo el planteamiento de la filosofía posterior a Descartes rechaza enfrentarse al problema del ser, puesto que, tal y como se entiende conforme a estas bases asentadas por el cartesianismo, el ser se mantiene extraño a este mundo del que sólo se perciben los entes, estando la cosa-en-sí ajena al sujeto. Al pretender que el ser posea las características de un ente cualquiera para que sea posible acceder a él como tal, se le buscan atributos y de ahí que la extensión como cosa corpórea tenga como atributo primero la propia extensión. Pero para Heidegger ello es un error que permite eludir el problema del ser. Heidegger lo expone cláramente:

Con lo dicho se han evidenciado los fundamentos ontológicos de la determinación del mundo como res extensa: esa determinación se basa en la idea de substancialidad, no sólo no aclarada en su sentido de ser, sino tenida por inaclarable, y expuesta mediante el rodeo a través de la característica substancial más importante de la substancia en cuestión (Ib., 94).

Lo ontológico no puede sustentarse en lo óntico. Descartes y todo el pensamiento que heredó sus ideas montan un entramado óntico-ontológico poco claro del término substancia que no tiene validez según nos lo presenta Heidegger, por lo que el problema sigue abierto.

6. El interés que Heidegger pone en rebatir las propuestas de Descartes parten de la pretensión de demostrar que lo que este último plantea con la ontología que va en el corazón de su filosofía es un sinsentido al confundir mundo con ente, marginando el fenómeno del mundo. Esto se debe a que la ontología planteada por Descartes no se pregunta en ningún momento acerca de la cuestión por el ser, sino que sólo se centra en lo ente, no siendo entonces ontología. Para Heidegger, Descartes se ha olvidado del mundo en su interpretación del mundo. En palabras de Heidegger

Descartes no sólo ofrece posiblemente una determinación ontológica errada del mundo, sino que su interpretación y los fundamentos de ella conducían a pasar por alto tanto el fenómeno del mundo como el ser del ente intramundano inmediátamente a la mano $(I b ., 95)$.

Así, el error cartesiano es confundir mundo con ente y no preocuparse por la comprensión de la entidad de ese ente denominado Dasein. El estudio cartesiano de la mismidad implica una inmersión de ésta en la realidad, pero en Descartes, esa mismidad se relacióna con un sujeto vinculado al mundo en tanto que puede conocerlo. Heidegger, por su parte, defiende que el vínculo del sí-mismo con el mundo ya está dentro de la propia constitución de la mismidad, de ese Dasein en tanto que éste está-en-el-mundo.

La crítica heideggeriana al planteamiento cartesiano no es sólo sobre el vínculo que plantea con la realidad, planteamiento de antemano equivocado al referirse a dicha realidad exclusivamente desde el conocer. También el modelo de conocimiento que de sus teorías surge, el racionalismo, se torna totalmente solipsista. Descartes defiende que el modo de acceder al mundo es mediante el conocimiento físico-matemático, instaurando así como pilar fundamental la labor científica en tanto que conocimiento seguro y fiable. Eso si, Descartes 
no es el único: también Kant y su interés por la física newtoniana. Esta fascinación por la ciencia y el conocimiento racional es objeto de la crítica de Heidegger. Y aunque el modelo cartesiano es superado por los planteamientos kantianos más tarde, éstos parten de que el conocimiento comienza en la sensación, la cual no es nada si no se pasa por el filtro de la razón (Kant, 1998).

Pero aún hay más. Si Kant elegirá como modelo de conocimiento la física newtoniana, en Descartes el modelo es, como señalamo, el modelo matemático euclidiano. Heidegger señala que "El conocimiento matemático es considerado como aquel modo de aprehensión del ente que pueda estar en todo momento cierto de poseer en forma segura el ser del ente aprehendido en él" (1977, 96). La elección de Descartes de las matemáticas se debe a su búsqueda de certezas, las cuales, según cree, dotarán de validez a su teoría. Pero una certeza no es lo mismo que una verdad, sino que aquella es siempre para el yo, por lo que tiene que ver más con el convencimiento que con el conocimiento. Así, Descartes instaura un conocimiento desde la óptica del yo, olvidándose luego de este yo al no saber salir del atolladero solipsista. Para salir del paso recurre a Dios, que está fuera del plano del mundo, buscando una especie de garantía de alteridad. Y con este retruécano se vincula la matemática con la teología, marginando a la ontología. La matemática denota lo que siempre es, lo que siempre permanece constante. "Propiamente es lo perdurantemente permanente. Lo que es tal lo conocen las matemáticas. Lo accesible en el ente por medio de ellas constituyen su ser" (Ib., 96). Mediante la matemática es posible acceder al ente, según plantea Descartes. Pero, tal y como lo plantea Heidegger, en esta sugerencia de la teoría cartesiana se halla el error fatal que hemos señalado:

Descartes no se deja dar el modo de ser del ente intramundano por éste mismo, sino que, básandose en una idea de ser no justificada y de origen no desvelado (ser=permanente estar-ahí), le prescribe, en cierto modo, al mundo su verdadero ser (Ib., 96).

Así, el recurso matemático de Descartes se centra en una explicación del ser como algo eterno e imperecedero, rechazando la temporalidad, base de la crítica heideggeriana. La explicación cartesiana no necesita recurrir a la sensación como objeto de conocimiento, ya que, para Descartes, todo en ella falla porque con el predominio de una ontología que vincula el ser con lo permanente, como sucedía en Aristóteles, se justifica el recurso a la razón y no a la percepción: "Los sentidos no hacen conocer en absoluto el ente en su ser, sino que sólo anuncian el carácter útil o perjudicial de las cosas externas del mundo para el ser humano corpóreo" (Ib., 96-97). Y por ello la crítica que Heidegger hace a Descartes ha de mostrarse implacable:

De esta manera, el análisis de las posibles vías de acceso al ente intramundano no cae, para Descartes, bajo el dominio de una idea de ser que ha sido tomada de una determinada región de este ente $(I b ., 97)$.

Al centrarse en una "parte" del ente, cae en el error de no contemplar al ser, considerando al Dasein como sustancia, estando entonces en la misma categoría que la extensión. 
La interpretación cartesiana de la res cogitans y de la res extensa toma la misma orientación ontológica que el resto de la tradición, esto es, mirando al mundo como algo ajeno.

Que tras Descartes se encubre el problema del mundo constituye una novedad, pero no creemos que sea una ruptura con la tradición al continuar el error existente sobre la pregunta por el ser, pregunta que a Heidegger le interesa recuperar como nuevo giro de la filosofía. La filosofía moderna se centra en valores añadidos, en atributos presupuestos pero que no dicen nada acerca del ser. "Los valores tienen en definitiva su origen ontológico únicamente en la previa posición de la realidad de la cosa como el estrato fundamental" (Ib., 99). Ello no logra la pretensión que persigue Heidegger:

Del mismo modo como Descartes no acierta, con la extensio como proprietas, en el ser de la sustancia, tampoco el recurso a las cualidades valiosas logra poner siquiera ante la vista el ser como estar-a-la-mano, y menos aun convertirlo en tema ontológico $(I b ., 100)$.

Con este error cartesiano se inaugura la Edad Moderna, la historia de un error. Por ello es necesario una correcta reinterpretación como la que Heidegger pretende llevar a cabo.

\section{Bibliografía}

Aristóteles (1957): Metaphysica, ed. W. Jäeger, Oxford University Press, Oxford.

Aristóteles (1996): Categories, ed. H. P. Cooke, Harvard University Press, Cambridge (Massachusset).

Descartes, René (1974): Ouvres, ed. C. Adam y P. Tannery, Cerf, París.

Heidegger, Martin (1977): Sein und Zeit. GB2, ed. F. W. von Herrmann. Klostermann, Frankfurt am Main. (Para la traducción de las citas al castellano empleamos la traducción de J. E. Rivera Cruchaga: (2004): Ser y tiempo, Trotta, Madrid).

Heidegger, Martin (1997): Nietzsche. GB6, ed. B. Schillbach, Klostermann, Frankfurt am Main.

Heidegger, Martin (2004): "Der Begriff der Zeit (Vortrag 1924)", en Der Begriff der Zeit. GB 64, ed. F.-W. von Herrmann, Klostermann, Frankfurt am Main.

Heidegger, Martin (2006): Zollikoner Seminare. Protokolle-Zwiegesprache-Briefe. ed. M. Boss, Klostermann, Frankfurt am Main.

Kant, Immanuel. (1998): Kritik der reinen Vernunft, Meiner Verlag, Hamburgo.

Moreno Márquez, César (2000): Fenomenología y filosofía existencial, Síntesis, Madrid.

Platón (1989): Phaedo, ed. J. Burnett, Oxford University Press. Oxford.

Ryle, Gilbert (2000): The concept of mind. Penguin, Londres. 